\title{
EFECTO DE TIPO DE TUTORES Y DENSIDAD DE SIEMBRA SOBRE EL RENDIMIENTO DE ÑAME ESPINO (Dioscorea rotundata Poir.)
}

\author{
Luna Castellanos, L. L. ${ }^{1 ;}$ Tamara Morelos, R. E. ${ }^{1}$ \\ \& Garcia Peña, J. A. ${ }^{2}$
}

\begin{abstract}
RESUMEN
El objetivo de la presente investigación fue determinar el efecto de tres tipos de tutores (tutor individual, espaldera simple y espaldera doble) y dos densidades de siembra (14,285 y 20,000 plantas/ha) sobre el rendimiento de Dioscorea rotundata cv brasilero. El experimento se realizó bajo un diseño de bloques completos al azar con arreglo en parcelas divididas con cuatro repeticiones. Las variables evaluadas fueron: rendimiento total de tubérculos $\left(\mathrm{t} \mathrm{ha}^{-1}\right)$, rendimiento de tubérculos comerciales, y adicionalmente se realizó un análisis económico de los tratamientos. Los resultados obtenidos mostraron diferencias altamente significativas para el factor densidad de siembra. Al emplear la densidad de 20,000 plantas/ha se obtuvieron los mayores rendimientos totales $(26,73$ t. $\left.\mathrm{ha}^{-1}\right)$ generando incrementos en $9,64 \%$ respecto a la menor densidad (14,285 plantas/ha) para esta variable. La utilización combinada de espaldera simple y la densidad de 20,000 plantas/ha generó aumentos de $9,3 \%$ en rendimiento de los tubérculos.
\end{abstract}

Palabras clave: Dioscorea, tubérculo, arreglo espacial, soporte individual, espaldera.

1.- Corporación Colombiana de Investigación Agropecuaria (AGROSAVIA). Sede El Carmen de Bolívar, Km. 1 vía Zambrano, Teléfono +57(1) 4227300 Ext. 2296. El Carmen de Bolívar-Bolívar, Colombia. Email: 1lunac@agrosavia.co, ORCID Luna Castellanos, L.: 0000000321727842; ORCID Tamara Morelos, R.: 0000000280579483.

2.- Corporación Colombiana de Investigación Agropecuaria (AGROSAVIA). Centro de investigación Turipaná. km 13 vía Montería-Cereté, Colombia. Telefono +57(1) 4227300 Ext. 225. Cerete, Córdoba, Colombia. ORCID García-Peña, J.: 0000000218059487.

Manuscrito recibido el 11 de julio de 2018 y aceptado para su publicación el 7 de diciembre de 2018.

Luna Castellanos, L. L.; Tamara Morelos, R. E. \& García Peña, J. A. Efecto de tipos de tutoresy densidad de siembra sobre el rendimiento de ñame espino (Dioscorea rotundata Poir.). FAVE - Ciencias Agrarias 17 (2): 7-21. CC BY-NC-SA 4.0 c) (i) (2) 


\section{ABSTRACT \\ Effect of type of tutors and population density on the yield of white yam (Dioscorea rotundata Poir.)}

The objective of the present investigation was to determine the effect of three types of tutors (individual tutor, simple espalier and double trellis) and two planting densities (14,285 and 20,000 plants/ha) on the performance of Dioscorea rotundata cv brasileiro. The experiment was carried out under a randomized complete blocks design with arrangement in divided plots with four repetitions. The evaluated variables were: total yield of tubers $(\mathrm{kg} / \mathrm{ha})$, yield of commercial tubers, and additionally an economic analysis of the treatments was carried out. The results obtained showed highly significant differences for the sowing density factor. When using the density of 20,000 plants / ha, the highest total yields $\left(26,73 \mathrm{t}^{\mathrm{h}} \mathrm{ha}^{-1}\right)$ were obtained, generating increases of $9.64 \%$ with respect to the lowest density (14,285 plants/ha) for this variable. The combined use of simple trellis and the density of 20,000 plants / ha generated increases of $9.3 \%$ in yield of the tubers.

Key words: Dioscorea, tubercle, spatial arrangement, individual support, espalier.

\section{INTRODUCCIÓN}

El tubérculo de ñame (Dioscorea sp.) constituye una de las principales fuentes alimenticias de muchas poblaciones en las regiones tropicales y subtropicales alrededor del mundo. Contiene importantes cantidades de carbohidratos, es fuente moderada de proteína y fibra $(4 ; 18 ; 28)$, características que hacen de este alimento indispensable para la seguridad alimentaria en los países de Asia, África y América.

En Colombia se produce el 22,06\% de los tubérculos de Dioscorea sp. que son demandados en muchos países de América, situándose como el segundo productor después de Haití $(23,71 \%)$ y Cuba $(21,54 \%)$ (11). Las áreas de cultivo se encuentran restringidas en su mayoría a la región Caribe con un 90\% de la producción nacional (25). Los departamentos de mayor producción de tubérculos de ñame: Bolívar $(44,10 \%)$, Córdoba $(34,87 \%)$ y Sucre $(7,26 \%)$ susten- tan la seguridad alimentaria y la generación de ingresos de los pequeños productores de esta región (2).

El cultivo de ñame espino en el Caribe colombiano, es producido en forma tradicional con escasa adopción de tecnología y prácticas de manejo. La baja competitividad del cultivo obedece principalmente a que los productores emplean bajas densidades de población (10,000 plantas/ha) para el establecimiento del cultivo, generando bajos rendimientos por unidad de área $(13,30$ t. ha-1), en relación con países productores de África con producciones de $25 \mathrm{t}$. ha ${ }^{-1}$ (23). Esto conlleva a la formación de tubérculos amorfos y de gran volumen que son rechazados por el mercado de exportación $(3 ; 25)$. Adicionalmente este cultivo requiere de la utilización de tutores de madera para brindar soporte durante el ciclo fenológico del cultivo. Esta práctica requiere que el agricultor disponga de grandes cantidades de estacas, lo cual genera incrementos del $20 \%$ en la mano de obra y los costos de pro- 
ducción del cultivo. Sumado a lo anterior, para la adquisición de estos soportes el agricultor dispone de la madera de linderos del predio o arbustos, lo cual incrementa los problemas de deforestación en la zona $(9 ; 16 ; 17)$.

Dentro de las alternativas de manejo integrado a implementar para mejorar los rendimientos del cultivo de ñame espino en la región caribe, el aumento de la densidad de población por unidad de área constituye una de las estrategias viables. A medida que aumenta el número de plantas, se mejora el aprovechamiento del espacio por los recursos ambientales, la alta competencia genera disminución en el peso y tamaño del tubérculo, aumenta el número de tubérculos/ha y mejora la forma (20). En segunda instancia, la utilización de sistemas de espalderas ayuda a orientar el crecimiento de la planta permitiendo que las hojas obtengan una buena distribución, mejoren el aprovechamiento de la radiación solar y la eficiencia fotosintética. La utilización de tutores proporciona incrementos de $19 \%$ a $10 \%$ en la masa media de los tubérculos de ñame, en relación con la ausencia de tutores, respectivamente (21). Por tal razón, en la presente investigación se determinó el efecto de tipos de tutores y densidad poblacional sobre el rendimiento de Diosocorea rotundata.

\section{MATERIALES Y MÉTODOS}

Localización: El presente estudio se llevó a cabo en la Corporación Colombiana de Investigación Agropecuaria (AGROSAVIA) sede El Carmen de Bolívar, ubicado geográficamente a $9^{\circ} 42^{\prime} 50.29^{\prime}$ ' $\mathrm{N}$ y $75^{\circ} 06^{\prime}$ 27.2" W en el municipio de Carmen de Bolívar (Bolívar, Colombia). Esta zona se caracteriza por presentar una precipitación anual promedio de $1100 \mathrm{~mm}$, humedad relativa del $76 \%$, temperatura promedio anual de $27,7^{\circ} \mathrm{C}$. El área pertenece a la zona climática cálido muy seco, a la formación de bosque seco tropical (BS-T). El experimento se desarrolló bajo condiciones de campo abierto, durante los meses de marzo a octubre de 2017. El suelo donde se instaló el ensayo presentó textura franco-arcillosa, $\mathrm{pH}=7,96 ; \mathrm{M} . \mathrm{O}=2,32 \% ; \mathrm{P}=79,71 \mathrm{mg} / \mathrm{kg} ;$ $\mathrm{Ca}^{2+}=28,71 \mathrm{Cmol} / \mathrm{kg} ; \mathrm{Mg}^{2+}=3,07 \mathrm{mg} / \mathrm{kg}$; $\mathrm{K}=0,63 \mathrm{mg} / \mathrm{kg} ; \mathrm{CIC}=34,36 \mathrm{Cmol} / \mathrm{kg}$.

Material vegetal: para el establecimiento del experimento se empleó el clon comercial de ñame espino (D. rotundata cv. Brasilero). A partir del picado de los tubérculos se obtuvieron minisemillas $(80-100 \mathrm{~g})$, las cuales fueron establecidas en bolsas negras (15 cm de alto x $15 \mathrm{~cm}$ de ancho), en cada una fue depositada una minisemilla. El sustrato empleado estuvo conformado por arena y tierra negra (proporción 1:1), este fue desinfectado mediante solarización y aplicación del fungicida Vitavax ${ }^{\circledR} 300$ (4g/l). La siembra de las minisemillas se realizó bajo condiciones protejidas empleando polisombra negra al $80 \%$, la producción bajo condiciones semicontroladas de luz y agua se realizó para garantizar la brotación completa y uniforme de las plantas. Una vez emergidas las plántulas, cuando alcanzaron una altura entre 15 y $20 \mathrm{~cm}$, fueron trasplantadas a las parcelas experimentales (18/05/2017).

Establecimiento del experimento: el suelo se preparó de forma mecánica realizando dos pases de cincel. Al momento del trasplante fueron adicionados en cada punto de siembra $80 \mathrm{~g}$ de materia orgánica y las plántulas fueron distribuidas con una dis- 
tancia fija entre calles de $1,0 \mathrm{~m}$ y distancia entre plantas de $0,5 \mathrm{~m}(20,000$ plantas $/ \mathrm{ha})$ y $0,7 \mathrm{~m}(14,285$ plantas/ha) respectivamente. Se aplicó riego por goteo teniendo en cuenta los requerimientos hídricos de la planta y la oferta ambiental de la zona. Se efectuaron labores de control químico y manual de malezas, amarre y guía de plantas, control fitosanitario de enfermedades y plagas. La fertilización del cultivo se realizó en forma de sulfato de amonio $\left(\mathrm{NH}_{4}\right)_{2} \mathrm{SO}_{4}$, con dosis de fertilización total de 25,80 g/planta. La aplicación se efectuó de forma fraccionada: $30 \%$ a los 30 días después de trasplante (DDT), y la segunda fracción del 70\% a los 60 DDT. Las dosis del fertilizante fueron calculadas teniendo en cuenta los requerimientos del cultivo y el análisis de suelo.

Diseño experimental: los tratamientos fueron distribuidos en un diseño experimental de bloques completos al azar, con arreglo en parcelas divididas, y cuatro repeticiones. La parcela principal correspondió al factor A: densidades de siembra $(20,000$ y 14,285 plantas/ha), con un área de $72 \mathrm{~m}^{2}$, en la subparcela se incluyó el factor B: tipo de tutores, tutor individual (tutor/planta), tutorado con sistema de espaldera/surco (espaldera simple) y espaldera doble (espaldera por cada dos surcos), con un área de $24 \mathrm{~m}^{2}$. Se establecieron en total 24 unidades experimentales.

Variables de respuesta: el rendimiento de los tubérculos se evaluó 7 meses después del trasplante, recolectando los tubérculos de los surcos centrales de cada parcela. Los tubérculos fueron clasificados en tres categorías: 1) tubérculos comerciales o tipo exportación, correspondiente a tubérculos de forma regular redondeada, libres de daños físicos o por plagas y enfermedades, con un peso entre 1,5-3,0 kg; 2) tubérculos no comerciales, los cuales se destinan al mercado local, correspondiente a tubérculos de forma irregular, con tamaño y peso superior o inferior al tipo exportación; 3) tubérculos de tercera categoría, los cuales no son aptos para la comercialización, corresponden a tubérculos de rechazo, pues presentan forma irregular, daños físicos o daños por plagas y enfermedades. Asimismo, se realizó un análisis económico de los tratamientos con la relación beneficio costo para cada tratamiento.

Análisis de datos: Los datos obtenidos fueron sometidos a pruebas de normalidad y homogeneidad de varianzas mediante las pruebas de Shapiro-Wilk y Levene respectivamente. Comprobados los supuestos se realizó un análisis de varianza y pruebas de comparación de medias de Tukey ( $p$ $\leq 0,05)$. Se utilizó el paquete estadístico S.A.S versión 9.2 (2008).

\section{RESULTADOS Y DISCUSIÓN}

Los resultados del análisis de varianza de los datos de rendimiento total (t. ha ${ }^{-1}$ ) indican que entre las densidades de siembra $(20,000$ y 14,285 plantas/ha) se presentaron diferencias altamente significativas $(\mathrm{p} \leq 0,01)$ (tabla 1). Para la densidad de 20,000 plantas/ha se obtuvieron los mayores rendimientos $\left(26,73 \mathrm{t}^{\text {t. ha }}{ }^{-1}\right)$ respecto a la densidad de 14,285 plantas/ha donde se alcanzaron 24,38 t. ha- ${ }^{-1}$, es decir, se obtuvieron 2,4 t. ha ${ }^{-1}$ de tubérculos menos en comparación con la mayor densidad de siembra empleada. Estos resultados coinciden con lo encontrado para Dioscorea rotundata por Opkara et al. (2014) (20), quie- 
nes evaluaron cinco densidades de siembra $(33,333 ; 40,000 ; 50,000 ; 66,666$ y 100,000 plantas/ha) en la temporada 2013-2014 en el cultivar de ñame espino "Adaka" y reportaron que el rendimiento de los tubérculos aumento con las poblaciones más altas (66,666 y 100,000 plantas/ha), el espaciamiento adoptado en estas densidades permitió que las plantas expresaran su potencial e interceptaran más radiación solar, favoreciendo la producción de foto asimilados y el llenado óptimo de los tubérculos. Por su parte, Cardona (2007) (6) al emplear $D$. rotundata cv. Guinea Negro obtuvo rendimientos de 50.7 t. ha ${ }^{-1}$ al emplear la densidad de siembra correspondiente a 28,604 plantas/ha, al emplear esta distancia se favoreció el aumento en peso de los tubérculos, no mostraron deformaciones y resultaron más pequeños y uniformes. Bajo las condiciones adecuadas este tipo de tubérculo facilitaría la cosecha mecánica, lo cual disminuirá costos de producción.

Resultados similares a los obtenidos en esta investigación han sido reportados por Rodríguez et al. (2001) (26) para Dioscorea alata accesión "6322", encontraron rendimientos de 27 t. ha ${ }^{-1}$ con una densidad de 22,500 plantas/ha, lograron obtener 21,000 tubérculos comerciales. ha ${ }^{-1}$. Estos

Tabla 1: Rendimiento total de tubérculos de Dioscorea rotundata bajo dos densidades de siembra y tres tipos de tutores.

\begin{tabular}{|c|c|}
\hline F. V & RTT (t. ha ${ }^{-1}$ ) \\
\hline \multicolumn{2}{|l|}{ A. Densidad } \\
\hline Densidad (14,285 plantas/ha) & 24,38 b \\
\hline Densidad (20,000 plantas/ha) & $26,73 \mathrm{a}$ \\
\hline Diferencia estadística & ** \\
\hline \multicolumn{2}{|l|}{ B. Tipo de tutorado } \\
\hline Tutor individual & 25,00 \\
\hline Tutor espaldera simple & 27,32 \\
\hline Tutor espaldera doble & 24,01 \\
\hline Diferencia estadística & n.s \\
\hline \multicolumn{2}{|l|}{ Interacción A x B } \\
\hline $\begin{array}{l}\text { Densidad (14,285 plantas/ha) x Tutor } \\
\text { individual }\end{array}$ & 25,53 \\
\hline $\begin{array}{l}\text { Densidad (14,285 plantas/ha) x Tutor } \\
\text { espaldera simple }\end{array}$ & 25,62 \\
\hline $\begin{array}{l}\text { Densidad (14,285 plantas/ha) x Tutor } \\
\text { espaldera doble }\end{array}$ & 21,72 \\
\hline $\begin{array}{l}\text { Densidad (20,000 plantas/ha) x Tutor } \\
\text { individual }\end{array}$ & 24,54 \\
\hline $\begin{array}{l}\text { Densidad }(20,000 \text { plantas/ha }) \times \text { Tutor } \\
\text { espaldera simple }\end{array}$ & 29,18 \\
\hline $\begin{array}{l}\text { Densidad }(20,000 \text { plantas } / \text { ha }) \times \text { Tutor } \\
\text { espaldera doble. }\end{array}$ & 26,45 \\
\hline Diferencia estadística & n.s \\
\hline C.V & $11.0 \%$ \\
\hline
\end{tabular}

F.V: fuente de variación; RTT: rendimiento total de tubérculos; *: Significativo ( $p \leq 0,05)$; n.s: No significativo; Medias con letras no comunes difieren estadisticamente según la prueba de Tukey para $\mathrm{p} \leq 0,05 ;$ C.V. Coeficiente de variación. 


\section{L. Luna Castellanos et al.}

resultados sugieren que la manipulación de la disposición espacial de las plantas empleada en esta investigación $(20,000$ plantas/ha) permitió obtener rendimientos análogos, siendo ventajosa esta alternativa ya que para la siembra se requiere menor cantidad de semillas, insumos, mano de obra que empleando 22,500 plantas/ha. Semaw (2014) (31) resalta que incrementos sustanciales en las densidades de siembra genera alta competencia entre las plantas de ñame espino, lo cual exige una mayor eficiencia en la utilización del recurso hídrico, la energía solar y los nutrientes del suelo. La respuesta positiva en incrementos en rendimiento por el aumento en la densidad de siembra depende del cultivar de ñame, la interacción con el ambiente y las condiciones ambientales.

Entre los tipos de tutores empleados en esta investigación no se encontraron diferencias significativas $(\mathrm{p} \geq 0,05)$ para la variable rendimiento total de tubérculos. Resultados similares han sido encontrados en investigaciones realizadas por Ennin et al. (2014) (9), quienes demostraron que la utilización de sistemas de espalderas comparados con los soportes individuales no genera incrementos significativos en crecimiento, rendimiento y numero de tubérculos de Dioscorea rotundata. Además, hallaron que el cultivo de ñame espino sin tutor reduce en un $32,5 \%$ los rendimientos. Tsado (2012) (32) encontró que en $D$. rotundata cv. Giwa el peso promedio del tubérculo obtenido bajo espalderas/surco realizadas con plástico de PVC arrojó 5,3 kg comparado con los palos de madera que fueron $3,4 \mathrm{~kg}$ mientras que el del control (sin estacas) fue de 1,4 kg. Los efectos obtenidos en este estudio son análogos a los reportados por Danquaq et al. (2014) (7) quienes hallaron que en $D$. rotundata cv Dente, el comportamiento de los rendimientos en dos zonas agroclimáticas de Ghana bajo diferentes tipos de soportes (soporte vivo/ planta y espalderas/surco) afectaron de manera significativa la biomasa foliar fresca y los rendimientos de los tubérculos. El uso de este tipo de sistemas de tutores en Ghana permitió reducir la deforestación de los árboles, los cuales eran utilizados para obtener estacas que dieran soportes al ñame. La utilización de espalderas en el cultivo de ñame es un sistema de orientación de la planta ecológicamente viable que permite mejorar la interceptación de luz. Los autores destacan que el cultivo de ñame sin tutor promueve el contacto del meristemo apical o la región meristemática de crecimiento con el suelo provocando quemaduras, esta condición afecta el desarrollo de la planta y consecuentemente la formación de los tubérculos. La realización de esta investigación ha permitido demostrar que la utilización de espalderas puede reemplazar el sistema tradicional de tutores/planta ya que se obtienen rendimientos similares, lo cual genera beneficios a los agricultores al disminuir mano de obra e insumos para el establecimiento del cultivo, además contribuye con la protección de los recursos vegetales.

La interacción entre los factores (densidad de siembra*tipo de tutor) no presentó diferencias significativas $(\mathrm{p} \geq 0,05)$ (tabla 1). Los rendimientos de los tubérculos en el cultivo de ñame espino no dependen de la combinación de la densidad de siembra y el tipo de tutor. Cada factor puede influenciar de manera independiente los rendimientos.

La prueba de comparación de medias para la variable rendimiento de tubérculos comerciales (Fig. 1), indica que el tipo de espaldera simple y tutor/planta constituyen los sistemas más eficientes para la produc- 
ción de ñame espino tipo exportación bajo las dos densidades de siembra evaluadas en esta investigación. El uso de soportes para D. rotundata representa incrementos del $65 \%$ en el rendimiento de los tubérculos comerciales (10). Los soportes pueden modificar de manera muy fuerte los procesos meteorológicos a escala micro climática, tales como el balance y distribución de la radiación solar dentro de la cobertura, el balance de energía, la intercepción y distribución del agua, la circulación del dióxido de carbono, humedad de suelo, aire y temperatura $(1 ; 15 ; 22 ; 27)$. La utilización del sistema de espaldera simple permite un mayor aprovechamiento de los recursos naturales (radiación solar) que a su vez ayuda a maximizar la producción de fotoasimilados que son aprovechados de manera óptima para el llenado del tubérculo $(5 ; 29)$.

El sistema de espaldera doble registró menores valores en el rendimiento de tubérculos comerciales para las dos densida- des de siembra evaluadas en comparación con el sistema de espaldera simple y tutor/ planta (figura 1). Estos resultados son similares a los reportados por Jiménez et al. (1992) (14), quienes indicaron que, entre los soportes convencionales, el tutor individual superó el tutor de barbacoa (espaldera doble). De Souza y Soares (1998) (8), Meneses (2008) (19) y Pérez et al. (2015) (24), reportan que la utilización de altas densidades de siembra y tutor de espaldera doble generan una alta competencia entre las plantas por la oferta ambiental, predomina un alto nivel de sombreado entre plantas vecinas, competencia por agua y nutrientes, que finalmente limita la fotosíntesis y partición de asimilados hacia el tubérculo.

Al emplear la densidad de siembra (14,285 plantas/ha) y espaldera simple se obtuvo una producción de $23,363 \mathrm{~kg}$. ha ${ }^{-1}$ que incremento en $2,3 \mathrm{~kg}$. $\mathrm{ha}^{-1}$ el rendimiento de los tubérculos comerciales, con respecto a la mayor densidad de siembra

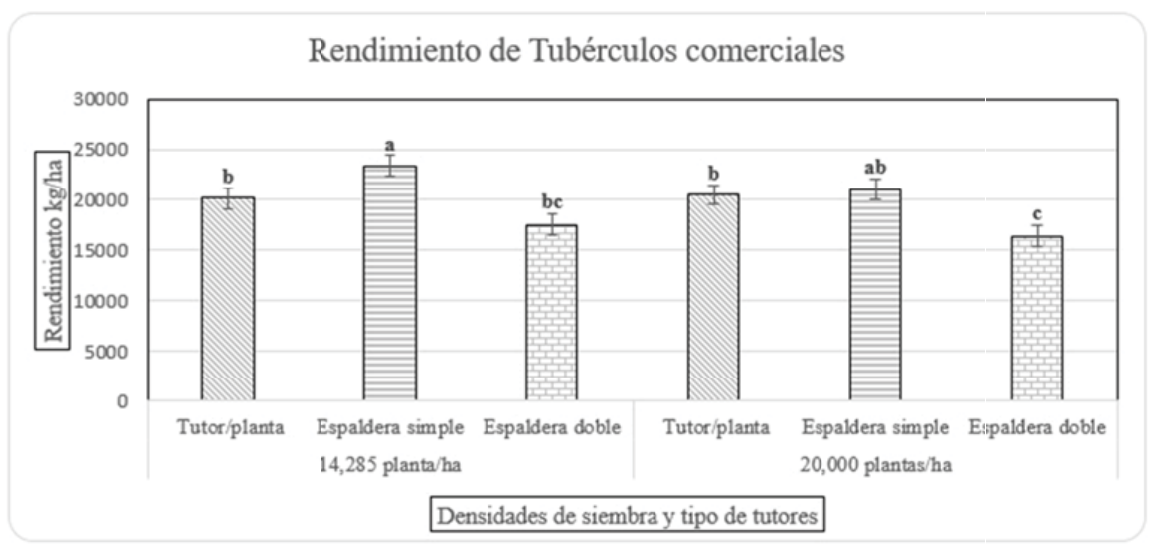

Fig. 1: Rendimiento de Tubérculos comerciales de ñame espino bajo efecto de dos densidades de siembra y tres tipos de tutores. Las barras de error representan el error estándar. Las letras rpreentan diferencias estadísticamente significativas $(p \geq 0,05)$. 
(20,000 plantas/ha) que permitió obtener $21,026 \mathrm{~kg}$. ha ${ }^{-1}$. Los resultados de esta investigación son semejantes a los obtenidos por Heredia et al. (2000) (13), donde alcanzaron incrementos en los rendimientos en 19,34\% al emplear la densidad de 16,000 plantas/ha, en relación con las producciones obtenidas con 12,000 plantas/ha, se infiere que los aumentos obtenidos con las mayores densidades fueron debidos al aumento en el número de plantas por unidad de área que por el peso individual de los tubérculos. Los tubérculos comerciales tipo exportación son el resultado de un mejor aprovechamiento de los recursos disponibles, favorecidos por una alta eficiencia durante su desarrollo, tal como indican Santos y Rodríguez (2008) (30) y Getachew (2012) (12).

\section{Análisis económico}

El comportamiento de los indicadores económicos para los factores evaluados se describe en la tabla 2, en la cual se observa que los ingresos brutos superan los costos totales en todas las combinaciones de los factores densidad*tipo de espaldera.

En el análisis económico de relación beneficio costo, se encontró que la densidad de 14,285 plantas/ha con los sistemas de espaldera simple y espaldera doble, presentaron relación beneficio costo de 1,8 . Por su parte, la densidad de 20,000 plantas/ha con espaldera simple y espaldera doble tuvo relación beneficio costo de 1,7; observándose una leve diferencia, lo que permite determinar que dado que los mayores rendimientos totales y de tubérculos comerciales se obtuvieron con la densidad de 20,000 plantas/ha y el sistema de espaldera simple, esta sería la mejor alternativa para el manejo del tutorado en ñame espino.

\section{CONCLUSIONES}

El desarrollo de esta investigación permitió establecer que al emplear altas densidades de siembra (20,000 plantas/ha) en el cultivo de ñame espino bajo las condiciones ambientales predominantes en el Carmen de Bolívar, Bolívar (Colombia) se obtuvieron incrementos significativos del 9,64\% en el rendimiento total de los tubérculos. La incorporación de sistemas de espalderas dentro de las estrategias de manejo integrado constituye una alternativa viable que permite mejorar los rendimientos del cultivo.

Tabla 2: Comportamiento de los indicadores económicos para los factores densidad de siembra y sistemas de espalderas.

\begin{tabular}{|l|c|c|c|c|}
\hline \multirow{2}{*}{ Indicador } & \multicolumn{2}{|c|}{$\begin{array}{c}\text { Densidad (14,285 } \\
\text { plantas/ha) }\end{array}$} & \multicolumn{2}{c|}{$\begin{array}{c}\text { Densidad (20,000 } \\
\text { plantas/ha) }\end{array}$} \\
\cline { 2 - 5 } & $\begin{array}{c}\text { Espaldera } \\
\text { simple }\end{array}$ & $\begin{array}{c}\text { Espaldera } \\
\text { doble }\end{array}$ & $\begin{array}{c}\text { Espaldera } \\
\text { simple }\end{array}$ & $\begin{array}{c}\text { Espaldera } \\
\text { doble }\end{array}$ \\
\hline Costo total (USD) & 4283.12 & 3511.84 & 5117.00 & 4344.26 \\
\hline $\begin{array}{l}\text { Ingreso bruto } \\
\text { (USD) }\end{array}$ & 7656.79 & 6362.88 & 8472.35 & 7247.62 \\
\hline Ingreso neto (USD) & 3373.67 & 2851.05 & 3355.35 & 2903.36 \\
\hline Beneficio-costo & 1,8 & 1,8 & 1,7 & 1,7 \\
\hline
\end{tabular}




\section{AGRADECIMIENTOS}

Esta investigación tuvo el apoyo financiero del Ministerio de Agricultura y Desarrollo Rural (MADR) de Colombia bajo el convenio TV-16. Al equipo de investigación de la Corporación Colombiana de investigación Agropecuaria (AGROSAVIA) sede El Carmen de Bolívar por el apoyo científico y logístico para el desarrollo de este proyecto.

\section{BIBLIOGRAFIA}

1.- ACOSTA, M. 2000. Manejo integrado de la antracnosis causada por Colletotrichum sp., en el cultivo de ñame (Dioscorea alata L.) Darién. IDIAP. Panamá. Plegable.

2.- AGRONET. 2018. Estadísticas área cosechada, producción y rendimiento de ñame, 2000-2016. Disponible en: http:// www. agronet.gov.co; consulta: enero de 2018.

3.- ÁLVAREZ, A. 2000. Prácticas agronómicas para el cultivo de ñame. pp. 33-39. En: Guzmán, M., Buitrago, G. (ed). Ñame: Producción de semillas por biotecnología. Editorial Universidad Nacional de Colombia, Bogotá.

4.- ALVIS, A.; VÉLEZ, C. Y RADA, M. 2008. Composición de ñames frescos cultivados en Colombia y sometidos a freído por inmersión. Información Tecnologica 19(1): 3-10.

5.- BUDELMAN, A. 1990. Woody legumes as live support systems in yam cultivation. Agroforestry systems 10(1): 47-59.

6.- CARDONA, J. 2007. Distancia de siembra en la producción y calidad de ñame Guinea negro (D. rotundata). The Journal of Agriculture of the University of Puerto Rico 91(1-2): 61-65.

7.- DANQUAH, E. O.; ENNIN, S. A.; LAMPTEY, J. N. Y ACHEAMPONG, P. P. 2014. Staking Options for Sustaina- ble Yam Production in Ghana. Sustainable Agriculture Research 4(1): 106- 113.

8.- DE SOUZA, L. Y SOARES, E. 1998. Estudo da freqüência de irrigação, densidade de plantas e níveis de nitrogênio na cultura do inhame. Revista Brasileira de Engenharia Agrícola e Ambiental 2(2): 37-41.

9.- ENNIN, S. A.; ISAAKA, R. N.; ACHEAMPONG, P. P.; NUMAFO, M. Y DANQUAH, E. 2014. Mechanization, Fertilization and Staking Options for Environmentally Sound Yam Production. African Journal Agricultural Research 9(29): 2222-2230.

10.- ENNIN, S. A.; OTOO, E. Y TETTEH, F. M. 2009. Ridging, a Mechanized Alternative to Mounding for Yam and Cassava Production. West African Journal of Applied Ecology 15 (1). 11.- FAO. 2018. Yam production statistics 2013. Faostat, FAO, Roma. Disponible en: http://www.fao.org/faostat/es/\#home; consulta: enero de 2018.

12.- GETACHEW, T.; BELEW, D. Y TULU, S. 2012. Yield and growth parameters of potato (Solanum tuberosum L.) as influenced by intra row spacing and time of earthing up: In Boneya Degem District, Central Highlands of Ethiopia. International Journal Agricultural Research 7(5): 255-265.

13.- HEREDIA, N.; CARMO, M. Y MINUZZI, A. 2000. Produção de cará (Dioscorea sp.) em diferentes densidades de plantio. Ciência e Agrotecnologia 24 (2):387-391

14.- JIMÉNEZ, J. M.; VÍQUEZ, E.; KASS, C. L. Y CHAVARRÍA, S. 1992. Uso de Erythrina berteroana y Gliricidia sepium como soportes vivos de ñame alado (Dioscorea alata L. cv 6322). El Chasqui. No. 29, p. 6-11.

15.- JIMÉNEZ, J.M. Y VARGAS, A. 1998. Soportes vivos para la producción de cultivos. In Apuntes de Clase del Curso Corto: Sistemas Agroforestales. Serie Técnica, Manual Técnico $\mathrm{N}^{\circ}$ 32. CATIE. Turrialba, CR. p279-288.

Revista FAVE - Ciencias Agrarias 17 (2) 2018 | 
16.- KUMAR, J. 2007. Lager Yam: In Underutilized and Underexploited Horticultural Crops. K.V Peter (ed.), New India Publishing Agency, New Delhi (India). Vol. 1, pp. 37-56.

17.- MANYONG, V.M.; IKPI, J.K.; OLAYEMI, S.A.; YUSUF, B.T.; OMONONA, V.; OKORUWA, F. Y IDACHABA, F.S. 2005.

Agriculture in Nigeria: identifying opportuinvestment. IITA, Ibadan, Nigeria. 159p.

18.- MARTIN, F.W. 1976. Tropical yams an their potencial: part 3. Dioscorea alata. U.S department of Agriculture. Agriculture Handbook $\mathrm{N}^{\circ}$ 495. P 40.

19.- MENESES, C. 2008. Absorção de NPK na cultura do inhame (Dioscorea cayennensis Lam) em três densidades de plantio. Tesis de maestría. Centro de Ciências Agrárias, Universidade Federal do Recôncavo da Bahia, Brasil.

20.- OKPARA, D., A.; IKORO, E.; MBAH Y AKPANINYANG, F. 2014. Growth and yield of white yam (Dioscorea rotundata Poir.) microsett in response to plant population and NPK fertilizer. Nigerian Journal Crop Science 2(1): 72-76.

21.- ONYEKA, T. J.; PETRO, D.; ANO, G.; ETIENNE, S. Y RUBENS, S. 2006. Resistance in water yam (Dioscorea alata) cultivars in the French West Indies to anthracnose disease based on tissue culture derived whole plant assay. Plant pathology 55(5): 671-678.

22.- OTOO, E.; ANCHIRINA, V. M.; ENNIN, S. A. Y ASIEDU, R. 2008. Sustainable yam production in Ghana the non-staking option. Journal of Food, Agriculture \& Environment 6(3-4): 391-396.

23.- PÉREZ, D. J. Y CAMPO, R. O. 2017. Efecto de la densidad poblacional sobre el rendimiento de ñame espino (Dioscorea rotundata Poir.) tipo exportación. Revista Colombiana de Ciencias Hortícolas 10(1): 89-98. nities for increased commercialization and

24.- PÉREZ, D.; CAMPO, R. Y JARMA, A. 2015. Respuesta fisiológica del ñame espino (Dioscorea rotundata Poir.) a las densidades de siembra. Revista de Ciencias Agrícolas 32(2): 117-124. 25.- REINA, Y. 2012. El cultivo de ñame en el Caribe colombiano. Documentos de Trabajo Sobre Economía Regional. No. 168. Banco de la República. 34 p.

26.- RODRÍGUEZ, W.; HILGER, T. H. Y LEIHNER, D. E. 2001. Effects of seed rates and plant populations on canopy dynamics and yield in the greater yam (Dioscorea alata L.). Field Crops Research 70(1): 15-26.

27.- ROJAS, M.; CONCEPCIÓN, J. Y VON CHON, K. 1997. Manejo integral del cultivo de ñame mecanizado (Dioscorea alata): Una opción para la producción sostenida. Modulo V Ñame, Yuca, Oteo. Divisa, Panamá. 44p.

28.- RUGCHATI, O. 2010. Comparasion in some characteristic of yam tubers starch (Dioscoraceae spp.) from Thailand. International Journal of environmental and Rural development 1-2. p102-106 29.- RUíZ, E. E. 2003. Severidad del complejo de enfermedades foliares en el cultivo de ñame (Dioscorea alata L.) en diferentes densidades de siembra y soportes vivos de madero negro (Gliricidia sepium (Jacq) Walp) y su rentabilidad en Azuero, Panamá.

30.- SANTOS, B. Y RODRÍGUEZ, P. 2008. Optimun in-row distances for potato minituber production. HortTechnology 18(3): 404-405.

31.- SEMAW, W. 2014. Effect of planting density on growth and yield of sweet potato (Ipomoea batatas (L.) Lam) varieties in Habru district, northern Ethiopia. College of Agriculture and Environmental Science, Haramaya University, East Harerge, Etiopia

32.- TSADO, E. K. 2012. Substituting wooden sticks with plastic stakes in yam production in Niger State, Nigeria. Journal of Natural Sciences Research 2(9): 88-96. 